\title{
Impact of estrogen-related receptor $\alpha$ on the biological characteristics of rat mandibular condylar chondrocytes
}

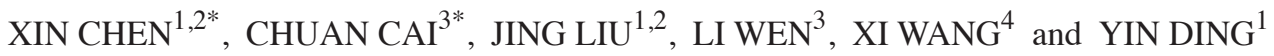 \\ ${ }^{1}$ Department of Orthodontics, School of Stomatology, the Fourth Military Medical University, Xi'an, Shaanxi 710032; \\ ${ }^{2}$ Department of General Dentistry, The $174^{\text {th }}$ Hospital of Chinese PLA, Xiamen, Fujian 361003; \\ ${ }^{3}$ Institute of Stomatology, Chinese PLA General Hospital, Beijing 100853; ${ }^{4}$ Department of Orthodontics, \\ The First Affiliated Hospital of Zhengzhou University, Zhengzhou 450052, P.R. China
}

Received December 12, 2013; Accepted March 19, 2014

DOI: $10.3892 / \mathrm{mmr} .2014 .2210$

\begin{abstract}
It is well-known that estrogen-related receptor $\alpha$ $(E R R \alpha)$ affects numerous metabolic pathways and biological functions in the body, although the function of ERR $\alpha$ in the mandibular condylar chondrocytes (MCCs) of the temporomandibular joint remains unclear. The aim of the present study was to investigate the effect of ERR $\alpha$ on the biological characteristics of MCCs in female rats. Immunofluorescent staining was used to observe the expression level and distribution of ERR $\alpha$ in MCCs and tissues. Quantitative polymerase chain reaction (qPCR) was performed to detect the impact of estrogen intervention on the biological characteristics of female rat MCCs and ERR $\alpha$ expression levels. Liposome transfection and XCT-790 were used to overexpress and inhibit ERR $\alpha$ expression, respectively, and then qPCR was performed to detect changes in the biological characteristics of MCCs. ERR $\alpha$ expression was detected in the nucleus and cytoplasm of rat MCCs. $17-\beta$ estradiol $(\mathrm{E} 2)\left(10^{-8} \mathrm{M}\right)$ increased the mRNA and protein expression levels of ERR $\alpha$, Sox9, GDF-5 and aromatase during in vitro MCC cultivation. In addition, E2 affected MCC proliferation through the regulation of ERR $\alpha$ expression levels. Overexpression of ERR $\alpha$ positively regulated the mRNA and protein expression levels of Sox9 and GDF-5, but did not exhibit a significant effect on the mRNA and protein expression levels of aromatase and Col2a1. In conclusion, ERR $\alpha$ exhibited an important regulatory role in the proliferation and differentiation of female Sprague-Dawley rat MCCs in vitro through regulating Sox9 and GDF-5.
\end{abstract}

Correspondence to: Professor Yin Ding, Department of Orthodontics, School of Stomatology, the Fourth Military Medical University, No. 169 Changle Road, Xi'an, Shaanxi 710032, P.R. China

E-mail: yindingcn@163.com

*Contributed equally

Key words: estrogen-related receptor $\alpha$, mandibular condylar chondrocyte, estrogen

\section{Introduction}

The mandibular condylar chondrocyte (MCC) is the key functional cell of the temporomandibular joint (TMJ). A number of TMJ diseases are caused by damage to these cells. Epidemiological studies have identified a difference in TMJ morbidity between male and female patients; TMJ morbidity in female patients is higher than in male patients and data indicate that changes in estrogen levels affect the metabolism of MCCs (1), suggesting that abnormal levels of estrogen may be associated with TMJ disease (2). A previous study, which generated different estrogen-level experimental environments and specifically regulated the expression of estrogen receptors (ERs), found that ER expression did not explain all phenomena observed in clinical and animal studies (3). This suggests that in addition to the ER classical signaling pathway, there may be other signaling pathways, with and without ER $\alpha$ association, involved in estrogen-related diseases.

Estrogen-related receptor $\alpha(E R R \alpha)$ is a type of orphan nuclear receptor that shares homology with ERs but without the binding of estrogen (4). In vitro studies have demonstrated that ERR $\alpha$ is expressed in the torso cartilage tissues of humans and animals, and is involved in a number of metabolic activities, including inflammation (5-10). The regulatory response of ERR $\alpha$ to estrogen may depend on the species, tissue and cell types $(11,12)$, and the expression and functions of ERR $\alpha$ in temporomandibular tissues and cells remain unclear.

In the present study, the ERR $\alpha$ expression levels in the MCCs of female Sprague-Dawley (SD) rats were investigated, to analyze the impact of estrogen on the biological characteristics of MCCs through the mediative effects of ERR $\alpha$. This may enable further understanding of MCC characteristics, as well as the mechanisms of physiological and pathological changes in MCCs.

\section{Materials and methods}

Primary cultivation and identification of MCCs. The present study was conducted in strict accordance with the recommendations of the Guide for the Care and Use of Laboratory Animals of the National Institutes of Health. The animal use protocol was reviewed and approved by the Institutional 
Animal Care and Use Committee of the Fourth Military Medical University (Xi'an, China). Six female SD rats (age, eight weeks), were sacrificed by cervical dislocation. The rats were then soaked in 75\% ethanol and MCCs were separated under sterile conditions. Serum-free RPMI-1640 medium (Sigma-Aldrich, St. Louis, MO, USA) was used to remove and rinse blood and other impurities adhered to the articular cartilage. The articular cartilage was then repeatedly cut with ophthalmic scissors to form sections of $\sim 1 \mathrm{~mm}^{3}$. A total of 2-3 $\mathrm{ml}$ of $0.25 \%$ trypsin solution (Sigma-Aldrich) was added for digestion in the incubator at $37^{\circ} \mathrm{C}$ for $20 \mathrm{~min} ; 3 \mathrm{ml}$ of $0.2 \%$ type II collagenase solution (Sigma) was then added and the mixture was further incubated for $2 \mathrm{~h}$. The cell suspension was pipetted, filtered using 200 mesh and centrifuged at $300 \mathrm{x} \mathrm{g}$ for $5 \mathrm{~min}$, and the supernatant was removed. RPMI-1640 medium supplemented with $10 \%$ fetal bovine serum was used to prepare the cell suspension. The cells were counted, then seeded and incubated at $37^{\circ} \mathrm{C}$, in $5 \% \mathrm{CO}_{2}$ and saturated humidity. The cells were then purified using the differential adhesion method (13). After $48 \mathrm{~h}$, the medium was replaced to remove any nonadherent cells. The remaining cells were cultivated for 3-5 days, then the medium was replaced with the common medium. When cell fusion had reached $80-90 \%$, passage was performed. The cell morphology was observed with an inverted microscope (Olympus, Tokyo, Japan),.

Immunofluorescent staining. The third generation of logarithmically-growing MCCs was prepared as a single cell suspension, then inoculated into the Lab-Tek II chamber slide (NUNC, Roskilde, Denmark) with density of $\sim 5 \times 10^{4}$ cells/chamber. The original culture medium was removed after $24 \mathrm{~h}$ conventional cultivation. Following washes with phosphate-buffered saline (PBS) solution for 3 times, $4 \%$ paraformaldehyde was added for fixation and $0.25 \%$ Triton $\mathrm{X}$ (Invitrogen Life Technologies, Carlsbad, CA, USA) was added for 15 -min incubation at $37^{\circ} \mathrm{C}$. After 15 min soaking in $3 \%$ $\mathrm{H}_{2} \mathrm{O}_{2}$, goat serum (Boster, Wuhan, China) was added to cover the cell-attached coverslip for blocking. The slide was then incubated for $30 \mathrm{~min}$ at $37^{\circ} \mathrm{C}$, followed by washing with PBS three times. Type II collagen polyclonal antibody (titer 1:100; Sigma-Aldrich) was added and the mixture was incubated for $2 \mathrm{~h}$ at $37^{\circ} \mathrm{C}$. PBS was used to replace the primary antibody in the negative control group. Following three washes with PBS, the secondary FITC-conjugated goat anti-mouse IgG (Beyotime, Nantong, China) was added in the dark and the slide was incubated for $1 \mathrm{~h}$ at $37^{\circ} \mathrm{C}$ then washed with PBS three times. The residue fluid was drained and DAPI was added for 15 min nuclear staining in the dark. Fluorescence microscopy (Olympus) was then used to observe and capture images of the staining situations.

Alcian blue staining. The MCCs were digested with $0.25 \%$ trypsin solution at $37^{\circ} \mathrm{C}$ for $3 \mathrm{~min}$, then seeded onto a 6-well plate and cultivated using the normal cultivation method. When the wall-adherent cells had grown to $80 \%$ confluence, the original culture medium was removed, the cells were washed with PBS buffer three times and $4 \%$ formaldehyde was used for fixation. The cells were again washed three times with PBS buffer, Alcian blue (Sigma) was added for 30 min staining and the cells were washed with water. PBS was used to replace
Table I. Primer sequences used in the reaction system.

\begin{tabular}{ll}
\hline Gene & \multicolumn{1}{c}{ Sequence } \\
\hline ERR $\alpha$ & F: 5'-GATGTGGCCTCTGGCTACCACTA-3' \\
& R: 5'-CGGACAGCTGTACTCGATGCTC-3' \\
Sox9 & F: 5'-TGAAGGGCTACGACTGGACG-3' \\
& R: 5'-ACTTGTAATCGGGGTGGTCTTT-3' \\
Col2a1 & F: 5'-TGGTGGAGCAGCAAGAGCA-3' \\
& R: 5'-CCCTCAGTGGACAGTAGACGGA-3' \\
GDF-5 & F: 5'-AGGGAGGTAACAGCAGCGT-3' \\
& R: 5'-CTCCAAGGCACTGATGTCAAAC-3' \\
Aromatase & F: 5'-CTTTTGAGACGATTCCAGGTGA-3' \\
& R:5'-GGATAAGTAATGCCCCAGAGTAGAT-3' \\
3-actin & F: 5'-GGAGATTACTGCCCTGGCTCCTA-3' \\
& R: 5'-GACTCATCGTACTCCTGCTTGCTG-3'
\end{tabular}

ERR $\alpha$, estrogen-related receptor $\alpha$; F, forward; R, reverse.

the Alcian blue in the negative control group. A solution of 95\% alcohol was used to dehydrate until the background was clear, then the cells were washed with water. The staining was then observed and recorded.

Immunocytochemical staining. The MCCs were seeded on coverslips at a density of $5 \times 10^{3}$ cells $/ \mathrm{ml}$ for $48 \mathrm{~h}$ and then fixed with cold acetone. Immunocytochemical analysis was performed using the streptavidin-biotin complex method according to the manufacturer's instructions (Zhongshan Golden Bridge Biotechnology Co. Ltd., Beijing, China). 3,3'-Diaminobenzidine (Boster) served as the chromogen. The primary antibody against ERR $\alpha$ was a monoclonal rabbit anti-human ERRa (ab41868; Abcam, Cambridge, UK) at a 1:100 dilution. For the negative control, the primary antibody was substituted with a commensurable volume of PBS. The samples were counterstained with hematoxylin and examined under an Olympus compound microscope (Olympus) equipped with a Nikon digital camera (Nikon, Tokyo, Japan).

$q P C R$. Third-generation MCCs were prepared as a cell suspension and inoculated into small culture flasks according to the grouping. To investigate the effects of E2 on ERR $\alpha$ and MCC-specific markers, MCCs were divided into an E2 group and control group. The MCCs in the E2 group were treated with $10^{-8}$ M 17- $\beta$ estradiol (E2; Sigma-Aldrich) for 48-h stimulation, while the E2 was replaced by an equivalent solvent (anhydrous ethanol) in the control group. To investigate the effects of overexpression and inhibition of ERR $\alpha$ on MCCs, the cells were transfected with the plasmid pcDNA3.1(+)-cont and pcDNA pcDNA3.1(+)-ERR $\alpha$, or treated with XCT790, respectively. After 48 h $10^{-8} \mathrm{M} 17-\beta$ estradiol stimulation, the MCCs were collected. The total RNA from MCCs was isolated using TRIzol reagent (Invitrogen Life Technologies), according to the manufacturer's instructions. Complementary DNA was synthesized from mRNA using Moloney murine leukemia virus reverse transcriptase (Invitrogen Life Technologies). qPCR was conducted with a Mastercycler EP Realplex4 (Eppendorf 
Table II. Sequences of the target fragmental primers.

$\begin{array}{ll}\text { Primer name } & \text { Primer sequence }\left(5^{\prime}-3^{\prime}\right)\end{array}$

$\begin{array}{ll}\text { ERR } \alpha-\mathrm{F} & \text { TTAGGTACCGCCACCATGTCCAGCCAGGTGGTGG } \\ \text { ERR } \alpha-\mathrm{R} & \text { CGGCGCTCGAGTCAGTCCATCATGGCCTCAAG }\end{array}$

ERR $\alpha$, estrogen-related receptor $\alpha$.

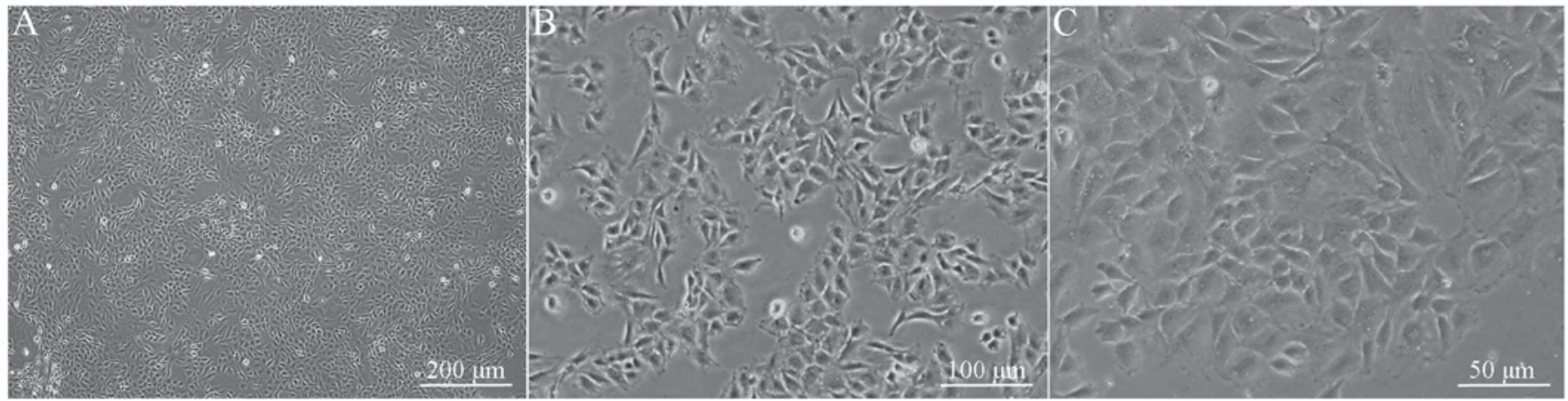

Figure 1. Cell morphology of the primary cultured rat mandibular condylar chondrocytes. Scale bar: (A) $200 \mu \mathrm{m}$; (B) $100 \mu \mathrm{m}$; and (C) $50 \mu \mathrm{m}$.

AG, Hamburg, Germany) and SYBR-Green (Invitrogen Life Technologies). The primer sequences used are shown in Table I. The reactions were performed under the following cycling conditions: $95^{\circ} \mathrm{C}$ for $10 \mathrm{~min}$, followed by 45 cycles of $95^{\circ} \mathrm{C}$ for $15 \mathrm{sec}$ and $60^{\circ} \mathrm{C}$ for $1 \mathrm{~min}$. The expression of the target genes was calculated using the formula $2^{-\Delta \Delta \mathrm{C} t}$. The expression data were normalized to the expression levels of the $\beta$-actin gene.

Western blotting. Briefly, cell extracts, containing $30 \mu \mathrm{g}$ total protein, were subjected to SDS-PAGE and then transferred to polyvinylidene fluoride membranes (Millipore, Billerica, MA, USA). The membranes were blocked and then probed with primary antibodies against ERR $\alpha$ (ab41868; Abcam), Sox9 (sc-20095; Santa Cruz Biotechnology, Inc., Santa Cruz, CA, USA), GDF-5 (sc-6901; Santa Cruz Biotechnology, Inc.), aromatase (sc-14245; Santa Cruz Biotechnology, Inc.), Col2al (ab34712; Abcam) and $\beta$-actin (ab8227; Abcam). The secondary antibodies, which were selected according to the species of origin of the primary antibodies, included mouse anti-goat IgG-HRP (A0216; Beyotime), goat anti-rabbit IgG-HRP (A0208; Beyotime), and donkey anti-goat IgG-HRP (A0181; Beyotime). Following incubation, the luminescent signals were detected using an enhanced chemiluminescence kit (Pierce, Rockford, IL, USA). The Quantity One software (Bio-Rad, Hercules, CA, USA) protein bands were used to measure the gray value of the target band corresponding to $\beta$-actin and the gray value of the ratio of the target protein in comparison with the strength of a sample index.

MCC proliferation detection. The experimental groupings were as follows: E2 $\left(10^{-8} \mathrm{M}\right)$ treatment group, XCT790 (5 $\mu \mathrm{M}$; Abcam) treatment group, E2 $\left(10^{-8} \mathrm{M}\right) / \mathrm{XCT} 790(5 \mu \mathrm{M})$ combined-treatment group and blank control group. The third generation of logarithmically-growing MCCs was prepared as a cell suspension, then inoculated into 96-well plates, with seeding density at $1 \times 10^{4}$ cells/well. This was performed 5 times for each treatment group. The cells were observed $24 \mathrm{~h}$ after inoculation; if the cells were adherent to the wall, the original culture medium was discarded. According to the instructions of the WST-1 kit (Boster), WST-1 solution was added to the cells for $2 \mathrm{~h}$-incubation at $37^{\circ} \mathrm{C}$ and $5 \% \mathrm{CO}_{2}$. The plate was then agitated for $1 \mathrm{~min}$ to fully mix the system for detection. The WST-1 treatment was performed on the cells at 48 and $72 \mathrm{~h}$, respectively, and then the relevant absorbance was determined using a spectrophotometer (BioPhotometer; Eppendorf). Absorbance was measured at $450 \mathrm{~nm}$.

Construction of vector and transfection. The target fragments were amplified using the primers listed in Table II and the pcDNA3.1(+) vector (Invitrogen Life Technologies) was then digested with restriction enzymes: HindIII at the upstream digestion site and EcoRI at the downstream digestion site. Following identification, the endotoxin plasmid was extracted and the vector was precipitated with ethanol. According to the manufacturer's instructions, Lipofectamine 2000 (Invitrogen Life Technologies) was used to transfect the overexpressed ERR $\alpha$; the transfection was performed when the third generation of logarithmically-growing MCCs had grown to 70-80\% confluence.

After $48 \mathrm{~h}$ treatment, the cells were collected for the detection of changes in MCC-related biological-characteristic markers. All experiments were repeated three times.

Statistical analysis. The collected experimental data were processed with SPSS 18.0 statistical software (SPSS, Inc., Chicago, IL, USA) and Student's t-test was used for intergroup comparisons. $\mathrm{P}<0.05$ was considered to indicate a statistically significant difference. 

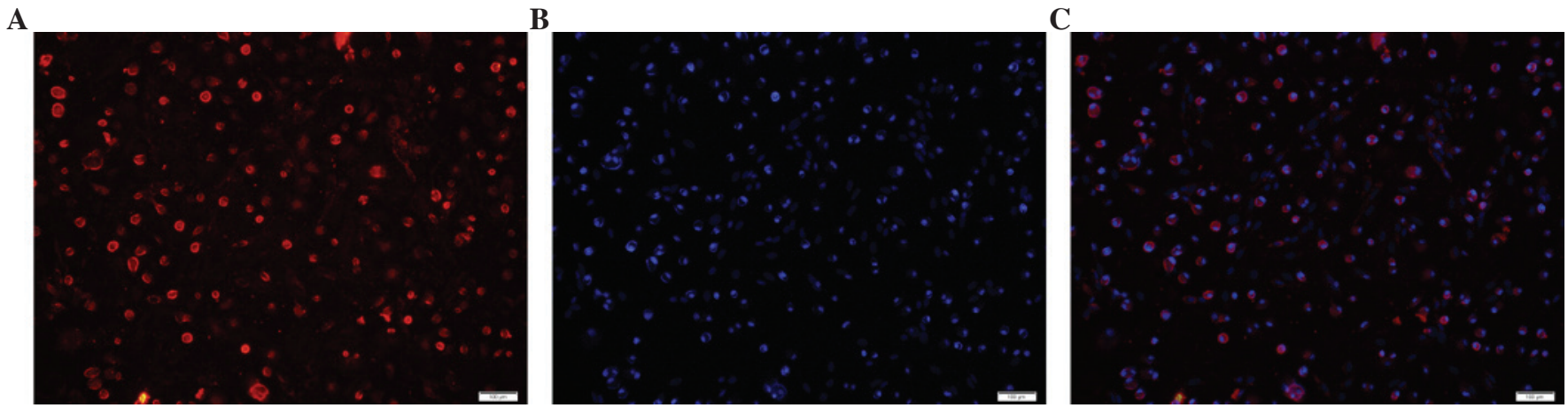

Figure 2. Immunofluorescent staining of collagen type II in rat mandibular condylar chondrocytes using phycoerythrin (PE) and DAPI fluorescence. (A) Type II collagen was widely expressed only in the cytoplasm demonstrated by PE fluorescence; (B) nuclei stained with DAPI; (C) merged PE and DAPI fluorescence. Scale bar, $100 \mu \mathrm{m}$.

A

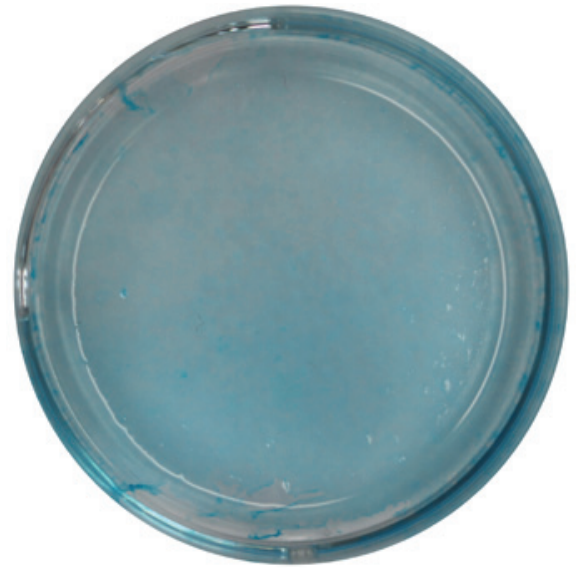

B

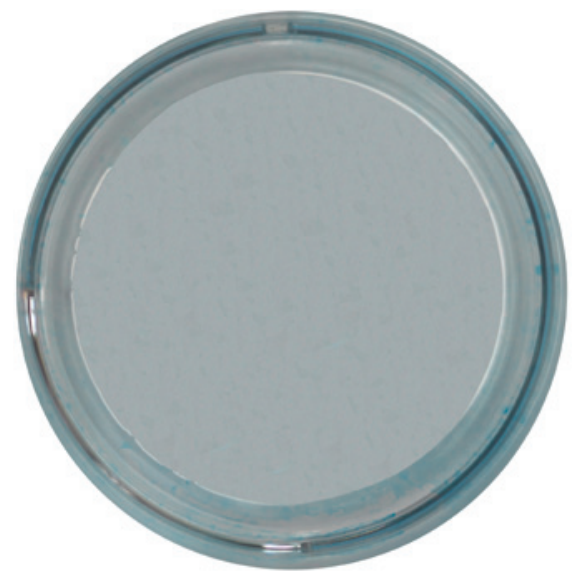

Figure 3. Alcian blue staining in rat mandibular condylar chondrocytes. (A) Positive Alcian blue staining of the third-passage mandibular condylar chondrocytes in normal culture medium. (B) Negative staining in the phosphate-buffered saline control group.

A

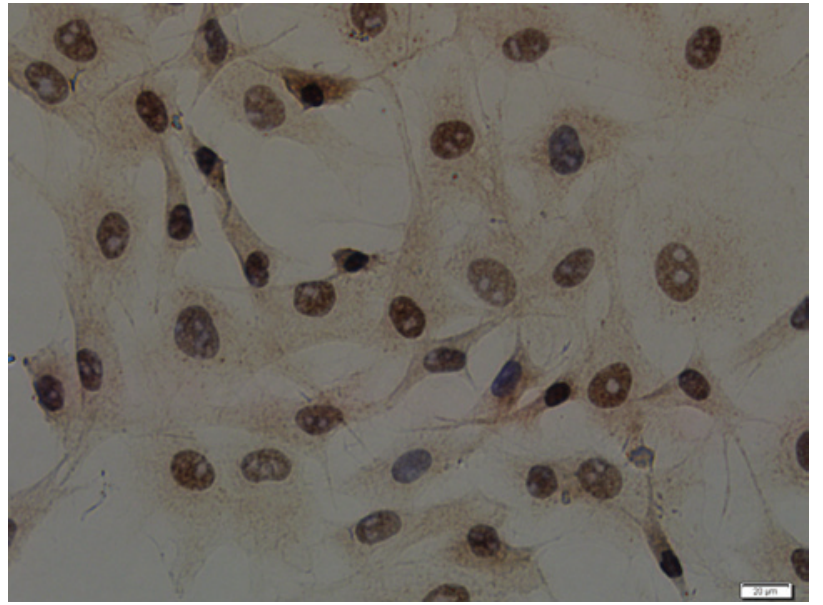

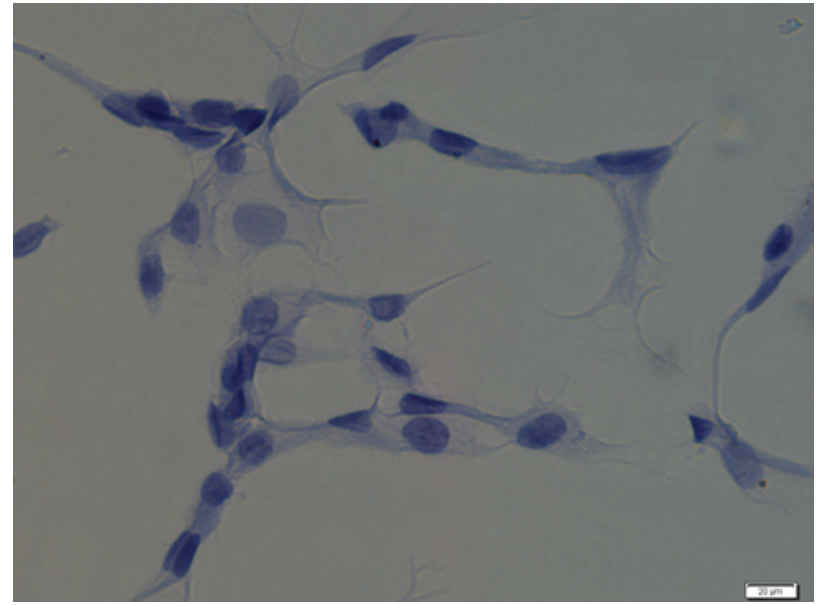

Figure 4. Immunocytochemical staining of estrogen-related receptor $\alpha$ (ERR $\alpha$ ) in rat mandibular condylar chondrocytes (MCC)s. (A) Positive staining of ERR $\alpha$ in the cytoplasm and nuclei of MCCs, as detected using anti-human ERR $\alpha$. (B) Negative immunoreactivity for ERR $\alpha$ in MCCs in the negative control group treated with phosphate-buffered saline. Scale bar, $20 \mu \mathrm{m}$.

\section{Results}

Characterization of MCCs. Observed under an inverted microscope, the primary cultured rat MCCs appeared spherical with a refractive extracellular matrix within $12 \mathrm{~h}$ and the cells were adherent to the wall within 12 to $48 \mathrm{~h}$. Following adherence, the cells stretched and exhibited polygon- or spindle-shaped morphology, with blunt cell bodies (Fig. 1). The cells aggregated in $\sim 1$ week, confluenced in $\sim 10$ days and formed a monolayer in $\sim 14$ days, and secreted large quan- 

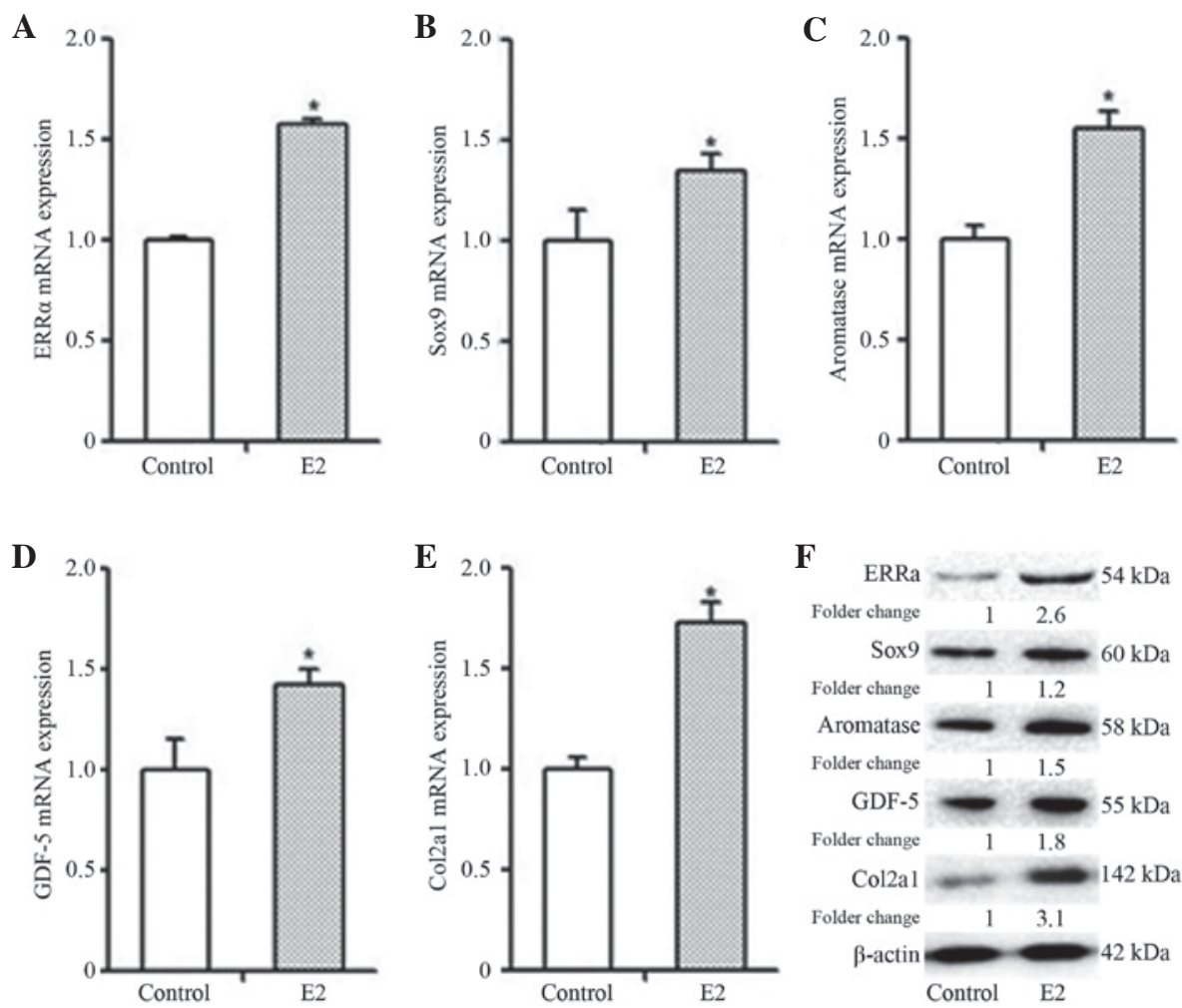

Figure 5. Changes in estrogen-related receptor $\alpha(\mathrm{ERR} \alpha)$ and mandibular condylar condrocyte (MCC)-specific marker expression levels after $48 \mathrm{~h} 17-\beta$ estradiol (E2) $\left(10^{-8} \mathrm{M}\right)$-simulated cultivation of MCCs. (A-E) Quantitative polymerase chain reaction detection results; (F) western blotting results. "P<0.05, vs. the control group.

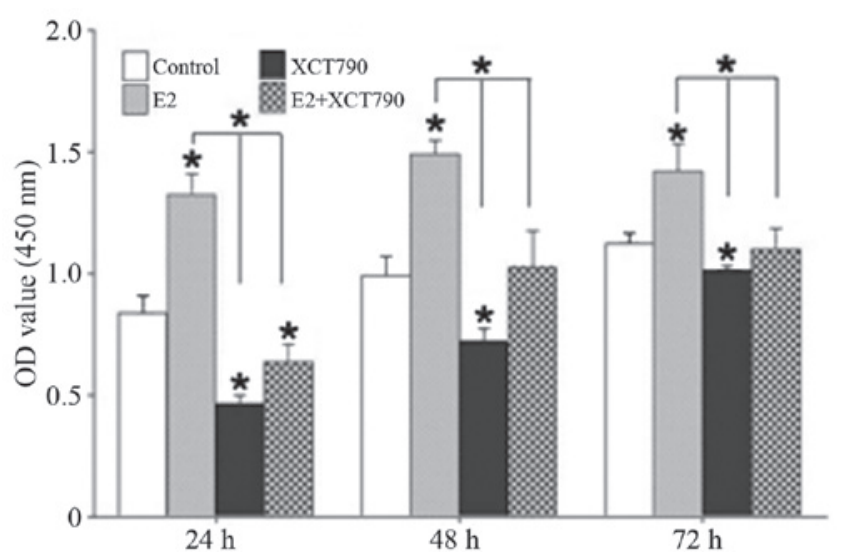

Figure 6. Impact of 17- $\beta$ estradiol (E2) and XCT790 treatment on mandibular condylar condrocyte proliferation, measured using WST-1 "P<0.05.

tities of refractive extracellular matrix. After three weeks, overlapping growth was observed in certain cells, with multilayer-clump-like growth zones in certain areas. The cells that exhibited positive immunofluorescent staining for cytoplasmic type II collagen accounted for $>90 \%$ cells in the random view field, while DAPI blue staining was observed, only in the nucleus (Fig. 2).

Alcian blue staining. Alcian blue staining revealed that the cultured cells were positively blue stained (Fig. 3A), exhibiting characteristics of cartilage-specific extracellular matrix, while the PBS control group exhibited no positive staining (Fig. 3B).
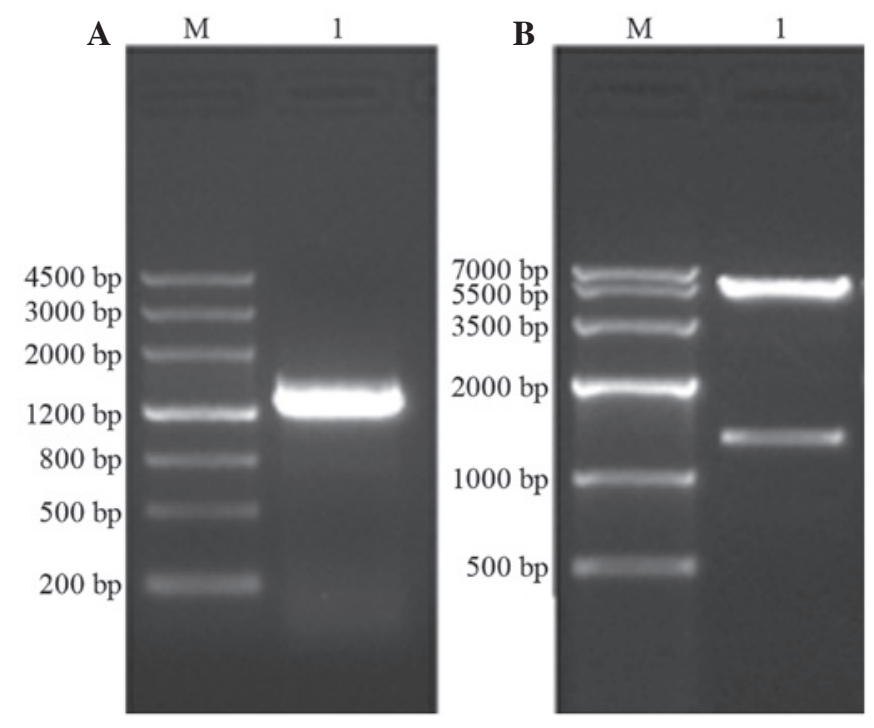

Figure 7. Agarose gel electrophoresis results. (A) Polymerase chain reaction products; lane 1, a band at 1,300 bp was observed, corresponding to estrogen-related receptor $\alpha$, ERR $\alpha$. (B) Recombinant plasmids following double-enzyme digestion; lane.1, pcDNA3.1 (+)-ERR $\alpha$. M, marker lane.

Expression of ERR a and MCC-related biological characteristic markers. As shown in Fig. 4A, ERR $\alpha$ was expressed in the MCCs; the nuclei and cytoplasm were brown-stained, although the expression in the nucleus was markedly greater. Fig. 4B shows the PBS negative control staining.

In order to investigate the impact of E2 on the biological characteristics of MCCs, different markers of MCC prolifera- 

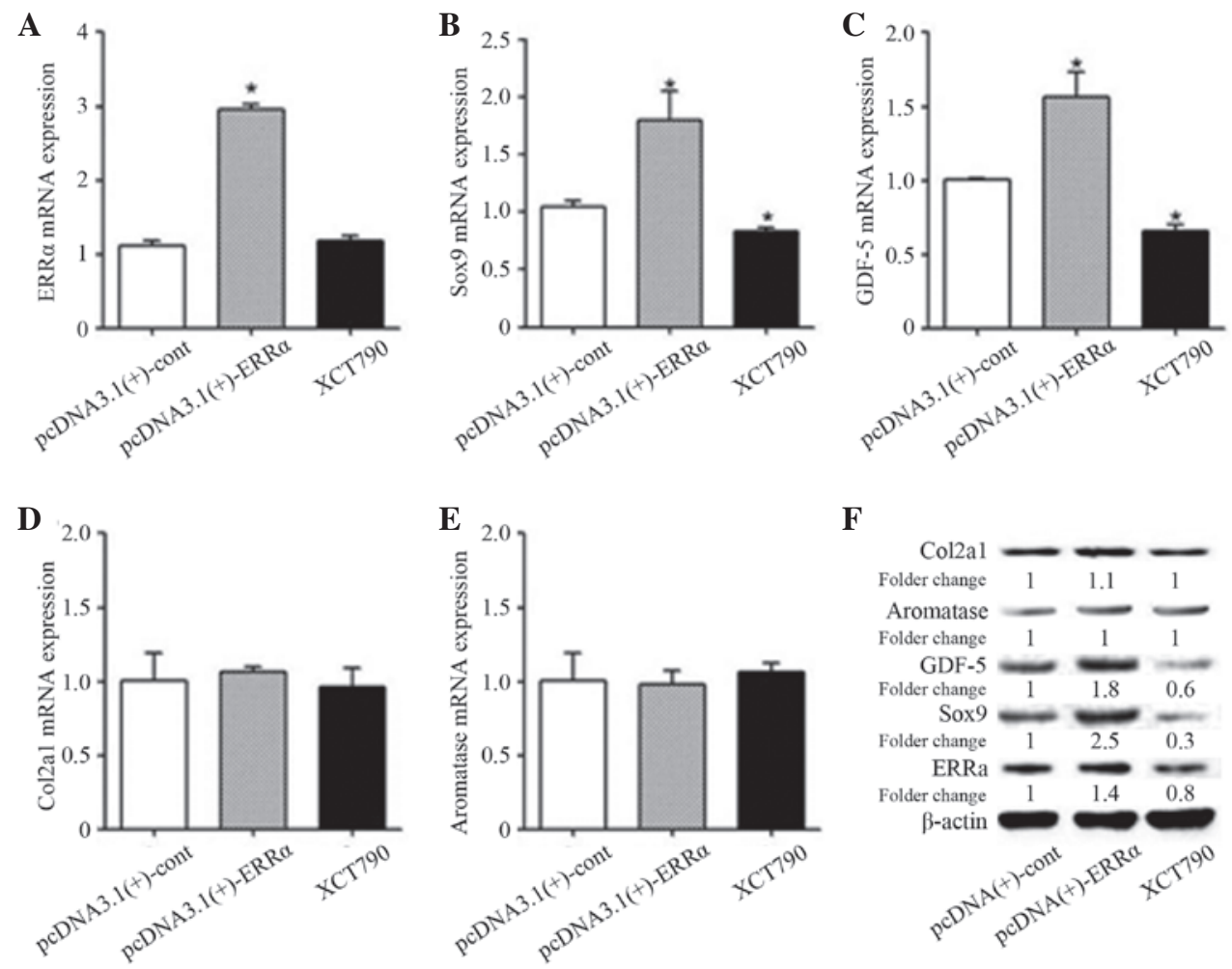

Figure 8. Transfected plasmid overexpressed estrogen-related receptor $\alpha(E R R \alpha)$; the expression levels of markers $48 \mathrm{~h}$ after XCT790 treatment of mandibular condylar chondrocytes (MCC)s. (A-E) mRNA expression changes. (F) Protein changes. "P<0.05, vs. the control group.

tion and differentiation were analyzed. Following E2 $\left(10^{-8} \mathrm{M}\right)$ stimulation for $48 \mathrm{~h}$, the mRNA and protein expression levels of ERR $\alpha$, Sox9, GDF-5, aromatase and Col2a1 in the MCC cells were significantly higher than those in the cells of the control group (Fig. 5; P<0.05). Therefore, E2 promoted the gene and protein expression of ERR $\alpha$, Sox9, GDF-5, aromatase and Col2al in the MCCs.

Effects of XCT790 on MCC proliferation. In order to reveal the impact of $\mathrm{E} 2$ on $\mathrm{MCC}$ proliferation and the possible regulatory effects of ERR $\alpha, \mathrm{E} 2\left(10^{-8} \mathrm{M}\right)$ and an inverse agonist of ERR $\alpha$, XCT790 $(5 \mu \mathrm{M})$, were used to treat the MCCs. WST-1 was performed at different time points to detect cell proliferation. Statistical analysis (Fig. 6) demonstrated that MCC proliferative capacity in the E2 $\left(10^{-8} \mathrm{M}\right)$ treatment group was higher than that in the control group and the E2 $\left(10^{-8} \mathrm{M}\right) / \mathrm{XCT} 790(5 \mu \mathrm{M})$ treatment group. In addition, the MCC proliferative capability of the XCT790 $(5 \mu \mathrm{M})$ treatment group was lower than that of the control group and the E2 $\left(10^{-8} \mathrm{M}\right)$ treatment group; and the MCC proliferative capability in the E2 $\left(10^{-8} \mathrm{M}\right) / \mathrm{XCT} 790(5 \mu \mathrm{M})$ treatment group was lower than that in the E2 $\left(10^{-8} \mathrm{M}\right)$ but higher than that in the XCT790 $(5 \mu \mathrm{M})$ treatment groups.

Construction of the ERRa eukaryotic expression vector. For electrophoretic identification of the PCR products, agarose gel electrophoresis was used to separate the samples and the $1,300 \mathrm{bp}$ band of ERR $\alpha$ was observed.

For the electrophoretic identification of recombinant plasmids, the recombinant plasmid pcDNA3.1 (+)-ERRaby was obtained following enzyme digestion and ligation reactions.
The minipreparation-extracted plasmids were double-enzyme digested and agarose gel electrophoresis was performed, which revealed two DNA bands (Fig. 7), one at $\sim 5.4 \mathrm{~kb}$, matching the size of the pcDNA3.1 (+) vector, and the other at $\sim 1,300 \mathrm{bp}$, matching the base pair number of ERR $\alpha$.

DNA sequence analysis of the recombinant plasmids. The sequencing results of pcDNA3.1-ERR $\alpha$ were performed using BLAST analysis following the correction. The nucleic acid sequences were consistent with the coding sequences of ESRRA in the GenBank database.

Expression levels of MCC-associated biological characteristic markers. Once ERR $\alpha$ overexpression in the transfected MCCs was confirmed, statistical analysis of the ERR $\alpha$ group compared with the control group revealed that the expression levels of the ERR $\alpha$ mRNA and protein were significantly increased $(\mathrm{P}<0.05)$ and the expression levels of Sox9 and GDF-5 mRNA and protein were also significantly increased $(\mathrm{P}<0.05)$. However, no significant difference was identified in the change in mRNA and protein expression levels of Col2al and aromatase (Fig. 8). The results showed that the transfection was successful, resulting in overexpression of ERR $\alpha$ mRNA and protein, and that ERR $\alpha$ overexpression exibited a positive regulatory role on the early proliferation and differentiation markers of MCCs, namely Sox9 and GDF-5, although showed no significant correlation with Col2a1 and aromatase, which were expressed by the mature MCCs.

In order to further clarify the role of ERR $\alpha$, XCT790 $(5 \mu \mathrm{M})$ was used to inhibit ERR $\alpha$ activity in MCCs. The changes in gene and protein expression levels of ERR $\alpha$, Sox9, 
GDF-5, Col2al and aromatase after $48 \mathrm{~h}$ were detected by qPCR and western blotting. The results revealed that compared with the control group, no significant difference was detected in the expression levels of ERR $\alpha$ mRNA in the XCT790 $(5 \mu \mathrm{M})$ treatment group, although the protein expression level was reduced. In addition, the mRNA and protein expression levels of Sox 9 and GDF-5 were reduced, but no significant changes were identified in the expression levels of Col2a1 and aromatase mRNA and protein. The results indicated that the inhibition of ERR $\alpha$ in MCCs with XCT790 (5 $\mu \mathrm{M})$ occurred mainly at the protein level, not at the gene level. In conclusion, these results suggest that XCT790 (5 $\mu \mathrm{M})$ inhibited the mRNA and protein expression of Sox 9 and GDF-5 through downregulation of ERR $\alpha$ protein expression.

\section{Discussion}

In the present study, the bodies of the primary MCCs were obtuse, appearing polygonal or spindle-like. Type II collagen immunofluorescent staining revealed that the positive staining cells covered $>90 \%$ of the area in the view field, indicating that the cultivated cells maintained the ability to synthesize type II collagen. The positive Alcian blue staining revealed that the cultured cells possessed the ability to secrete proteoglycans, thus, exhibiting the phenotype of MCCs $(14,15)$.

A study demonstrated that the expression of ERR $\alpha$ in tissues and cells is important with regard to the metabolic activities and biological functions of the body (16). ERR $\alpha$ is expressed in the bone and cartilage of the limbs and trunk, and may mediate the regulation of bone metabolism balance $(17,18)$. The TMJ is involved in speech, chewing and other functions (19). Due to its special structures, the characteristics of the TMJ are different from that of other joints in the trunk and limbs. MCCs are an important component of TMJ, which undergoes constant change to respond to the external stress stimuli and internal hormone levels. To the best of our knowledge, the present study was the first study to reveal positive ERR $\alpha$ expression in the MCCs. ERR $\alpha$ expression in the nuclei and cytoplasm of MCCs further suggests that ERR $\alpha$ may act as a transcription factor, shuttling between the nucleus and cytoplasm, and actively regulating signal transduction in cells.

Estrogen exhibits extensive effects on body metabolism, and is closely associated with cell proliferation, differentiation and maturation (20). An increasing number of studies have demonstrated that estrogen is important in the metabolism of bone/cartilage tissue, and that its biological effects are associated with the estrogen dose and response pathways (21-25). The imbalance in estrogen regulation depends largely on the changes in its response pathways. In addition to the traditional ER signaling pathway, there are a number of associated by-pathways. Thus, to investigate the impact of E2 on the biological characteristics of MCCs and ERR $\alpha$ expression, the physiological concentration of E2 $\left(10^{-8} \mathrm{M}\right)(26)$ was used in the present study to process in vitro cultured MCCs. The mRNA and protein expression levels of ERR $\alpha$, Sox9, GDF-5, aromatase and Col2a1, were elevated following stimulation of MCCs by E2 $\left(10^{-8} \mathrm{M}\right)$ for $48 \mathrm{~h}$, indicating that the physiological concentration of $\mathrm{E} 2$ exhibited the capacity to promote early proliferation and differentiation, and late maturation of in vitro-cultured MCCs.
Considering that the ERR $\alpha$ expression levels increased in the MCCs following E2 $\left(10^{-8} \mathrm{M}\right)$ treatment and that ERR $\alpha$ has been reported to promote cell proliferation (27), the physiological concentration of E2 and the specific ERR $\alpha$ inverse agonist, XCT790, were used in the present study to treat the MCCs, and WST-1 was used to observe the changes in cell proliferation. The WST-1 results revealed that E2 promoted the proliferation of MCCs and that when XCT790 (5 $\mu \mathrm{M})$ was used to inhibit ERR $\alpha$ expression, the proliferative capacity of the MCCs was reduced. Therefore, ERR $\alpha$ promoted MCC proliferation and mediated the E2-induced promotion of cell proliferation. The results suggest that the ERR $\alpha$ expression in MCCs was directly or indirectly influenced by E2, and that ERR $\alpha$ mediated the signal transduction through which E2 affected MCC proliferation, differentiation and maturation, although the specific signaling pathway for these effects remains unclear.

ERR $\alpha$ has been confirmed to regulate different metabolic activities of humans and multiple animals under different conditions, and is crucial in controlling metabolic balance (28). In the present study, following plasmid transfection to overexpress ERR $\alpha$, the gene and protein expression levels of Sox 9 and GDF-5 were found to significantly increase. The gene and protein expression of Col2al and aromatase increased marginally, although not significantly. In the XCT790 treatment group, ERR $\alpha$ protein expression, but not mRNA expression, was significantly inhibited, consistent with the results of a study of the mechanism of XCT790 inhibition of ERR $\alpha$ (29). Following XCT790 treatment, the gene and protein expression levels of Sox 9 and GDF-5 declined along with the reduction in ERR $\alpha$ protein expression levels, although no significant changes were identified in the gene and protein expression levels of Col2al and aromatase.

In MCCs, the observation that changes in ERR $\alpha$ protein expression levels may increase or reduce mRNA and protein expression levels of Sox 9 and GDF-5 suggested that Sox 9 and GDF- 5 were regulated by ERR $\alpha$, and that these genes were downstream responsive genes. Activation of the Sox 9 gene may promote proliferation and aggregation of cartilage precursor cells, and further differentiation towards chondrocytes (30). The changes in Sox9 expression levels were consistent with those of ERR $\alpha$, indicating that ERR $\alpha$ may be a direct regulator of Sox 9 in MCCs. In addition, ERR $\alpha$ affected the signal response of cell proliferation and accumulation in the early stages of MCC metabolic change. GDF-5 is the predominant regulator of cartilage growth and differentiation, and it was also positively regulated by ERR $\alpha$. In the early stages of bone/cartilage metabolism, changes in the expression levels of Sox 9 may induce changes in the subsequent bone/cartilage metabolism (17). Whether the effects of ERR $\alpha$ on GDF-5 were mediated through Sox 9 remains to be confirmed by further studies.

Col2al and aromatase are expressed by mature cartilage, and are important in secreting type II collagen and topically synthesizing estrogen to maintain the stable chondrocytes function. Col2a1 and aromatase expression levels were not sensitive to ERR $\alpha$ regulation, further suggesting that the effect of ERR $\alpha$ on MCCs is mainly reflected in upper-end regulation, namely proliferation, differentiation and maturation. This does not exclude the possibility that ERR $\alpha$ may exert indirect effects 
on Col2al and aromatase. As the course of cell proliferation, differentiation, maturation and apoptosis is a continuous metabolic process, all stages influence each other; the associations are close rather than isolated. In order to maintain normal biological functions, the balance of cell proliferation, differentiation and maturation are precisely regulated by hormone levels, transcription factors and numerous other factors in the body (31). The interactions among ERR $\alpha$ and other transcription factors remain to be elucidated.

In conclusion, ERR $\alpha$ was identified as an important regulator in the early proliferation and differentiation of MCCs, positively regulating Sox9. Overexpressed ERR $\alpha$ promoted proliferation of MCCs, although the impact on cell maturation is unclear. ERR $\alpha$ expression may be affected by multiple factors, including estrogen levels. Further studies regarding the ERR $\alpha$-mediated biological characteristic changes of MCCs may aid further understanding of the impact of changes in the body estrogen levels on the physiological and pathological changes in the TMJ.

\section{References}

1. Chander CL and Desa FM: The effects of estrogens on cartilage degradation using in vivo and in vitro models. Agents Actions 34: 282-284, 1991.

2. Meisler JG: Chronic pain conditions in women. J Womens Health 8: 313-320, 1999.

3. Sims NA, Dupont S, Krust A, Clement-Lacroix P, Minet D, Resche-Rigon M, Gaillard-Kelly M and Baron R: Deletion of estrogen receptors reveals a regulatory role for estrogen receptors-beta in bone remodeling in females but not in males. Bone 30: 18-25, 2002

4. Giguère V, Yang N, Segui $P$ and Evans RM: Identification of a new class of steroid hormone receptors. Nature 331: 91-94, 1988.

5. Bonnelye E and Aubin JE: Estrogen receptor-related receptor alpha: a mediator of estrogen response in bone. J Clin Endocrinol Metab 90: 3115-3121, 2005.

6. Bonnelye E, Zirngibl RA, Jurdic P and Aubin JE: The orphan nuclear estrogen receptor-related receptor-alpha regulates cartilage formation in vitro: implication of Sox9. Endocrinology 148: 1195-1205, 2007.

7. Fujimoto J and Sato E: Clinical implication of estrogen-related receptor (ERR) expression in uterine endometrial cancers. J Steroid Biochem Mol Biol 116: 71-75, 2009.

8. Sonoda J,Laganière J, Mehl IR, et al: Nuclear receptor ERR alpha and coactivator PGC-1 beta are effectors of IFN-gamma-induced host defense. Genes Dev 21: 1909-1920, 2007.

9. Bonnelye E, Saltel F, Chabadel A, Zirngibl RA, Aubin JE and Jurdic P: Involvement of the orphan nuclear estrogen receptor-related receptor alpha in osteoclast adhesion and transmigration. J Mol Endocrinol 45: 365-377, 2010.

10. Rajalin AM, Pollock H and Aarnisalo P: ERRalpha regulates osteoblastic and adipogenic differentiation of mouse bone marrow mesenchymal stem cells. Biochem Biophys Res Commun 396: 477-482, 2010.

11. Liu D, Zhang Z, Gladwell W and Teng CT: Estrogen stimulates estrogen-related receptor alpha gene expression through conserved hormone response elements. Endocrinology 144: 4894-4904, 2003.
12. Shigeta H, Zuo W, Yang N, DiAugustine R and Teng CT: The mouse estrogen receptor-related orphan receptor alpha 1: molecular cloning and estrogen responsiveness. J Mol Endocrinol 19: 299-309, 1997.

13. Khan IM, Bishop JC, Gilbert S and Archer CW: Clonal chondroprogenitors maintain telomerase activity and Sox9 expression during extended monolayer culture and retain chondrogenic potential. Osteoarthritis Cartilage 17: 518-528, 2009.

14. Lin Z, Willers $\mathrm{C}, \mathrm{Xu} \mathrm{J}$ and Zheng MH: The chondrocyte: biology and clinical application. Tissue Eng 12: 1971-1984, 2006.

15. Schulze M, Kuettner KE and Cole AA: Adult human chondrocytes in alginate culture. Preservation of the phenotype for further use in transplantation models. Orthopade 29: 100-106, 2000 (In German).

16. Lu D, Kiriyama Y, Lee KY and Giguère V: Transcriptional regulation of the estrogen-inducible $\mathrm{pS} 2$ breast cancer marker gene by the ERR family of orphan nuclear receptors. Cancer Res 61: 6755-6761, 2001

17. Bonnelye E, Reboul P, Duval N, Cardelli M and Aubin JE: Estrogen receptor-related receptor alpha regulation by interleukin-1beta in prostaglandin E(2)- and cAMP-dependent pathways in osteoarthritic chondrocytes. Arthritis Rheum 63: 2374-2384, 2011.

18. Gallet $M$ and Vanacker JM: ERR receptors as potential targets in osteoporosis. Trends Endocrinol Metab 21: 637-641, 2010.

19. Pirttiniemi P, Kantomaa T and Sorsa T: Effect of decreased loading on the metabolic activity of the mandibular condylar cartilage in the rat. Eur J Orthod 26: 1-5, 2004.

20. Gruber CJ, Tschugguel W, Schneeberger C and Huber JC: Production and actions of estrogens. N Engl J Med 346: 340-352, 2002.

21. Jakob F, Ebert R, Ignatius A, Matsushita T, Watanabe Y, Groll $\mathrm{J}$ and Walles $\mathrm{H}$ : Bone tissue engineering in osteoporosis. Maturitas 75: 118-124, 2013.

22. Bay-Jensen AC, Slagboom E, Chen-An P, Alexandersen P, Qvist P, Christiansen C, Meulenbelt I and Karsdal MA: Role of hormones in cartilage and joint metabolism: understanding an unhealthy metabolic phenotype in osteoarthritis. Menopause 20: 578-586, 2013.

23. Martín-Millán M and Castañeda S: Estrogens, osteoarthritis and inflammation. Joint Bone Spine 80: 368-373, 2013.

24. Lee HR, Kim TH and Choi KC: Functions and physiological roles of two types of estrogen receptors, $\mathrm{ER} \alpha$ and $\mathrm{ER} \beta$, identified by estrogen receptor knockout mouse. Lab Anim Res 28: 71-76, 2012.

25. Orajärvi M, Puijola E, Yu SB, Liu X, Tiilikainen P, Wang M, Raustia A and Pirttiniemi P: Effect of estrogen and dietary loading on condylar cartilage. J Orofac Pain 26: 328-336, 2012.

26. Yasuoka T, Nakashima M, Okuda T and Tatematsu N: Effect of estrogen replacement on temporomandibular joint remodeling in ovariectomized rats. J Oral Maxillofac Surg 58: 189-197, 2000.

27. Bianco S, Lanvin O, Tribollet V, Macari C, North S and Vanacker JM: Modulating estrogen receptor-related receptor-alpha activity inhibits cell proliferation. J Biol Chem 284: 23286-23292, 2009.

28. Ranhotra HS: The estrogen-related receptor alpha: the oldest, yet an energetic orphan with robust biological functions. J Recept Signal Transduct Res 30: 193-205, 2010.

29. Lanvin O, Bianco S, Kersual N, Chalbos D and Vanacker JM: Potentiation of ICI182,780 (Fulvestrant)-induced estrogen receptor-alpha degradation by the estrogen receptor-related receptor-alpha inverse agonist XCT790. J Biol Chem 282: 28328-28334, 2007.

30. Zhou G, Zheng Q, Engin F, et al: Dominance of SOX9 function over RUNX2 during skeletogenesis. Proc Natl Acad Sci USA 103: 19004-19009, 2006.

31. Desvergne B, Michalik L and Wahli W: Transcriptional regulation of metabolism. Physiol Rev 86: 465-514, 2006. 